\title{
3D Rotational angiography (3DRA) one year's local experience
}

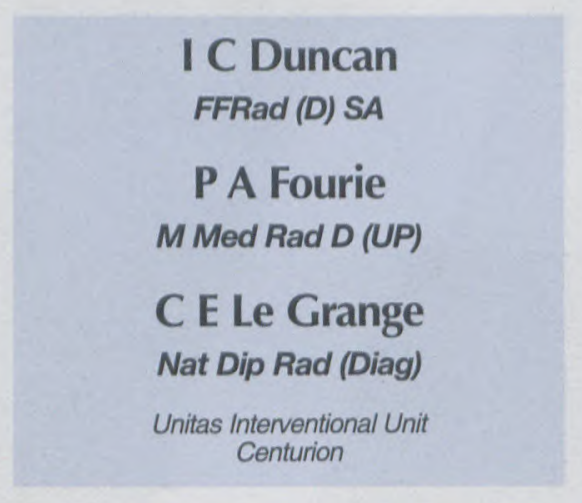

\section{Introduction}

Three-dimensional rotational angiography (3DRA) can trace its roots back to a 1975 paper by Voight $e t$ al.' describing rotational cerebral roentgenography. Rotational angiography itself had been shown to improve the diagnostic yield and accuracy of angiographic studies of cerebral aneurysms. ${ }^{2,3}$ The hardware required later for 3DRA thus already existed by the mid-to-late 1990s when this rotational angiographic capability was fused with ultrafast powerful modern computers to create the $3 \mathrm{D}$ computed rotational angiograms we see today. The earliest reports concerning the clinical use of this modality were published between 1996 and $1998 .^{46}$ Once established, many modern angiographic machines were adapted to perform the 3DRA function, involving some hardware changes mainly to existing $\mathrm{C}$-arm gantries and motors, and incorpora- tion of an ultrafast computer and relevant software package for data processing and manipulation. This new modality caught on rapidly in the neurointerventional fraternity and very soon further reports concerning the clinical value of 3DRA began to appear in the literature. ${ }^{7-10}$ Its major role in the pre-treatment imaging of cerebral aneurysms was quickly established and this remains its primary function to this day, with many major neurointerventional centres throughout the world now having 3DRA capabilities.

\section{DRA in South Africa}

The Unitas Interventional Unit was founded in July 1999. It employs a Phillips V5000 Integris monoplane digital angiography unit initially provided with standard rotational angiographic capabilities. Shortly after opening the unit we began to see computer-generated images produced using the data acquired during rotational acquisitions on other modified angiographic machines overseas and also read the first reports in the literature concerning the uses and benefits of 3DRA in neurodiagnostic and neurointerventional applications. ${ }^{46}$ This convinced us that we would ultimately require such capabilities in our own unit if we were to provide a level of diagnostic and interventional expertise on par with the best overseas. At a cost of over one million Rand and with little hope of ever directly recuperating this initial capital outlay by way of additional billing, we added the 3D package to our angiographic unit in early January 2002. A strong counter-argument against installing this package at the time was that CT and MR angiographic reconstructions were supposedly becoming good enough to provide the same information via noninvasive methods. ${ }^{11}$ Despite the high quality of 3D reconstructions created from CT and MR data nowadays, the level of detail contained within an angiographic reconstruction is clearly superior to that demonstrated by other means.

\section{How it works}

Many radiologists will probably be aware of the feature of standard rotational digital angiography. Here a precisely counterbalanced $\mathrm{C}$-arm rotates around the patient in a $180^{\circ}$ arc over a period of $7-8$ seconds $(<5$ seconds in newer machines) while contrast is being injected into the target vessels usually at a rate of between $3-5 \mathrm{ml} / \mathrm{s}$ for cranial or similar vessels. One hundred contrast-enhanced images are obtained in this process (12.5 frames/s). The digital data thus acquired represent a continuous volume of information similar to a volumetric CT scan. The angiographic image, however, is collimated to the field of view determined, in turn, by the image intensifier. This can vary from as little as $8 \mathrm{~cm}$ to as much as $40 \mathrm{~cm}$ depending upon the size of the image intensifier itself and its magnification capabilities. After acquisition, this continuous volume of digital data 


\section{ORIGINAL ARTICLE}

is fed to an ultrafast computer which recreates a usable three-dimensional image, presented on a workstation which allows a real-time interactive interface with the dataset. Electronic manipulation of the images allows rotation, magnification, translation and cutting of the images (Fig. 1). Prerequisites for good image quality include absolutely no patient movement during image acquisition, and that the contrast is injected at a high enough flow rate and volume to give optimal vessel opacification, usually by means of a dedicated power injector. The contrast resolution of the

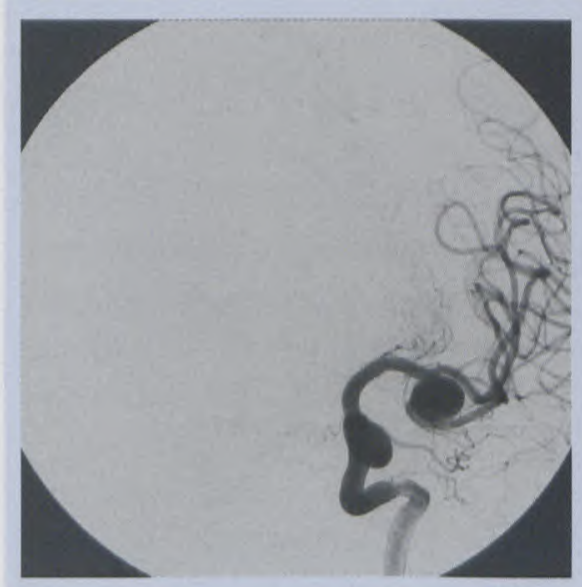

Fig. 1a. Selective left internal carotid digital subtraction arteriogram (DSA) showing a large left middle cerebral bifurcation aneurysm.

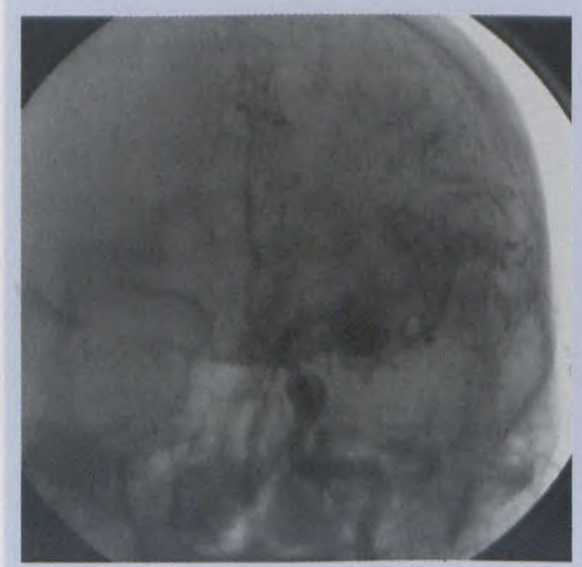

Fig.1b. A non-subtraction digital rotational angiogram is obtained.

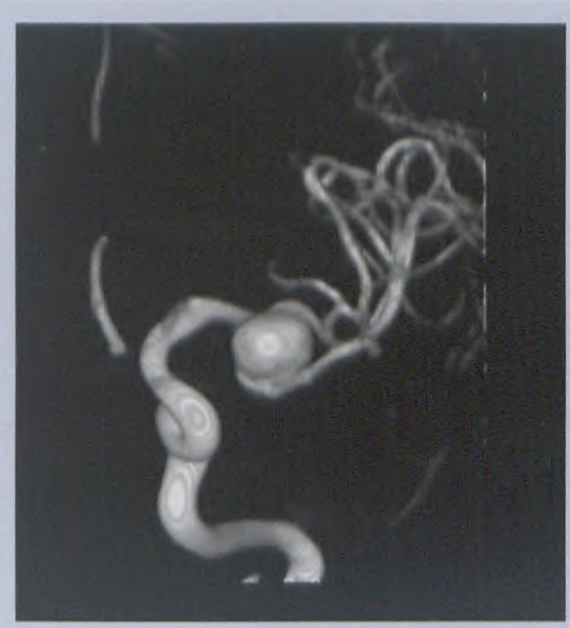

Fig. 1c. A first reconstruction is performed by the computer showing a volume-rendered image of the entire field of view. The windows are adjusted to show the contrast-filled vessels only.

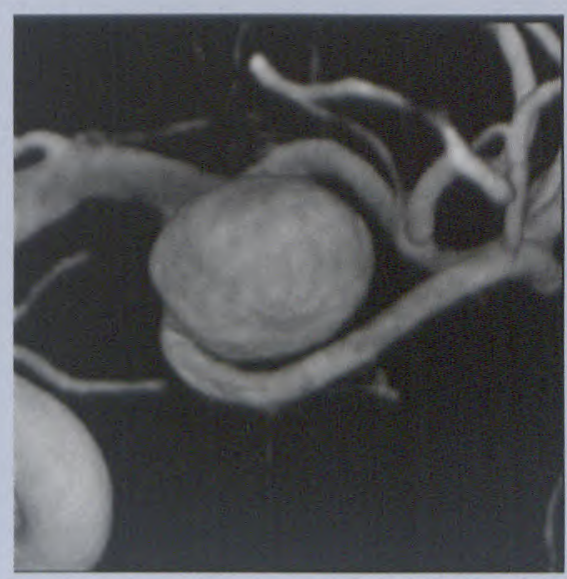

Fig. 1d. A second more detailed reconstruction is obtained showing a smaller field of view but more detailed image.

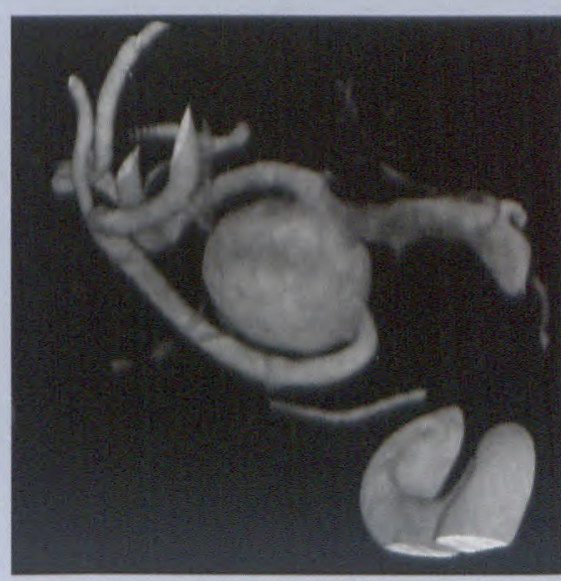

Fig. 1e. The image can be rotated in any direction and can be visualised from behind as seen here.

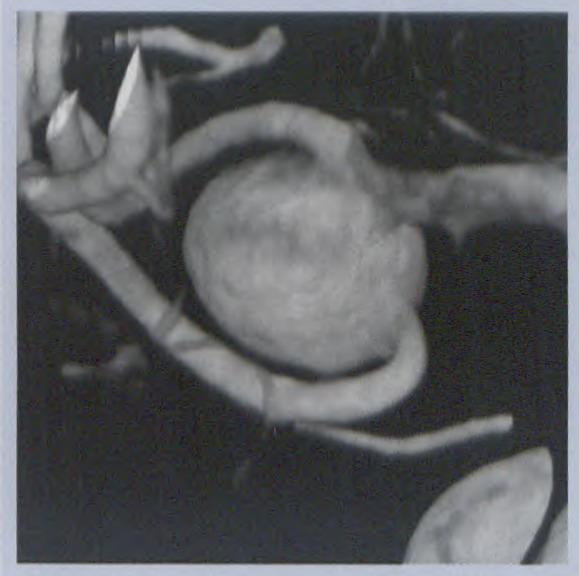

Fig. If. The image can be 'zoomed' or reduced as required.

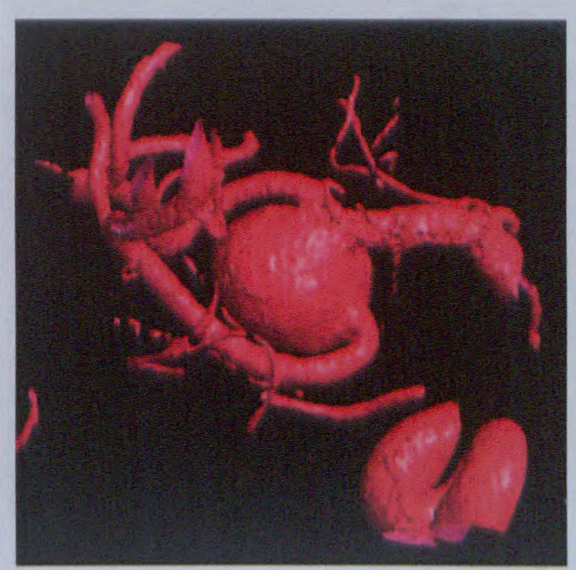

Fig.1g. The image can be displayed as a surface shaded display.

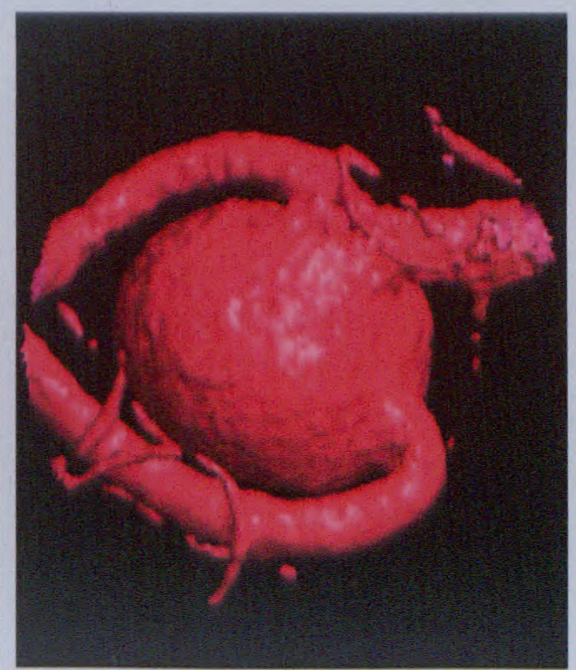

Fig. 1h. The image can be further cropped to remove adjacent unwanted information. 


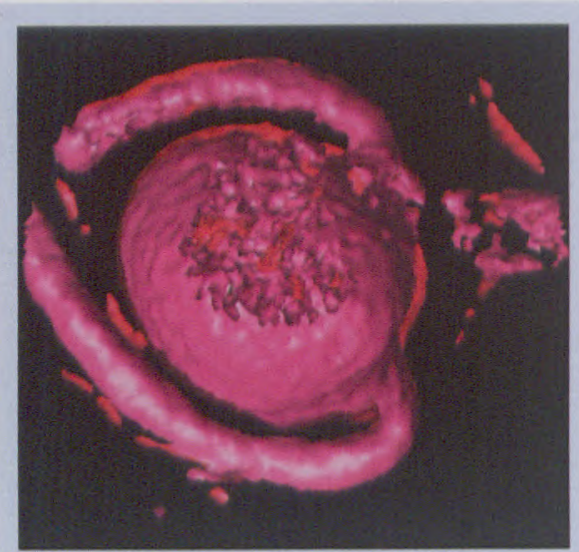

Fig. 1i. The image can be 'cut open' to reveal the internal architecture of the aneurysm.

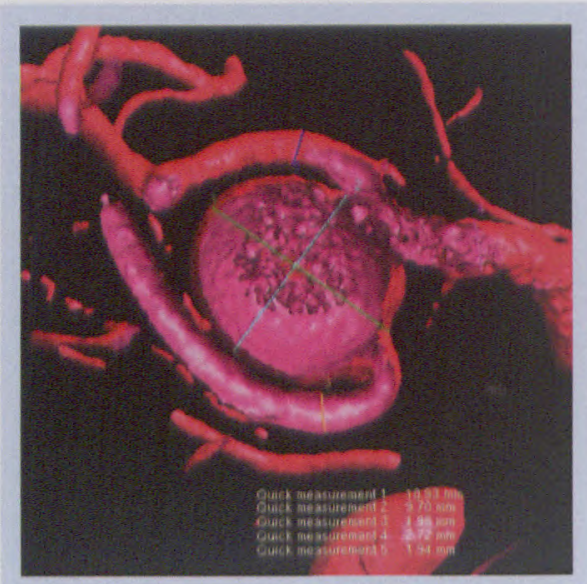

Fig. 1j. Measurements, accurate to within $2 \%$, can be made without the need for external calibration.

image depends upon the rate and volume of the contrast injection. Avoidance of patient movement during acquisition often necessitates the use of a general anaesthetic in order to optimise the quality of the study. The risks of an anaesthetic must be weighed up against the risks of multiple, repeated catheterisations and contrast injections in a restless or nervous patient, and increased radiation accumulation whilst trying to find an optimal projection in order to see a particular aspect of a lesion adequately, such as the neck of a cerebral aneurysm. One must remember that there is also considerable risk attached to an inadequate or even missed diagnosis, such as not seeing a small recently ruptured cerebral aneurysm due to the acquisition of images in an inadequate number of projections, as we have occasionally seen as well. A small percentage of our patients who had 3DRA studies while awake developed a transient ischaemic episode during acquisition due to the 8 second contrast injection time used. No permanent neurological sequelae have occurred in any of our patients to date as a result of these acquisitions. We generally now anaethetise all patients in whom we anticipate the performance of a 3DRA from the onset. This also allows us to acquire the images during suspended respiration to further limit movement artefact if required. The initial data acquisition is a non-subtracted rotational angiogram. Removal of unwanted bony and other detail from the image is not achieved by subtraction but rather by manipulation of the window levels and widths leaving only the high density image of the contrast within the blood vessels. The images obtained with 3DRA are never degraded by the presence of metallic items such as coils, clips or stents (Fig. 2). The creation of a mask is not necessary unless there is an object requiring subtraction from the image such as coils within an aneurysm lumen or surgical aneurysm clips that may obscure part of the contrast-filled lumen or aneurysm neck.

\section{Post-processing and image storage}

Once in the computer the data are reconstructed to give the $3 \mathrm{D}$ image. This can be displayed in one of several ways, namely volume-rendered, maximum-intensity projection or surface-shaded display images. The

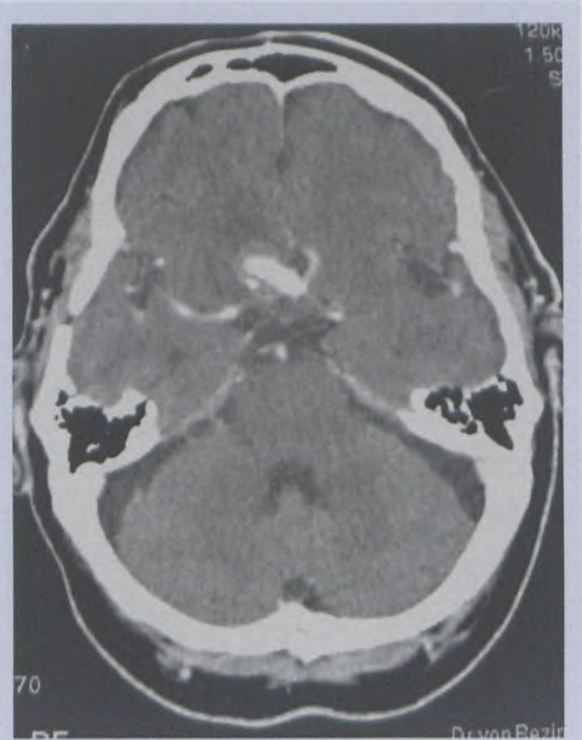

Fig. 2a. Axial enhanced CT image shows an aneurysm related to the anterior communicating artery.

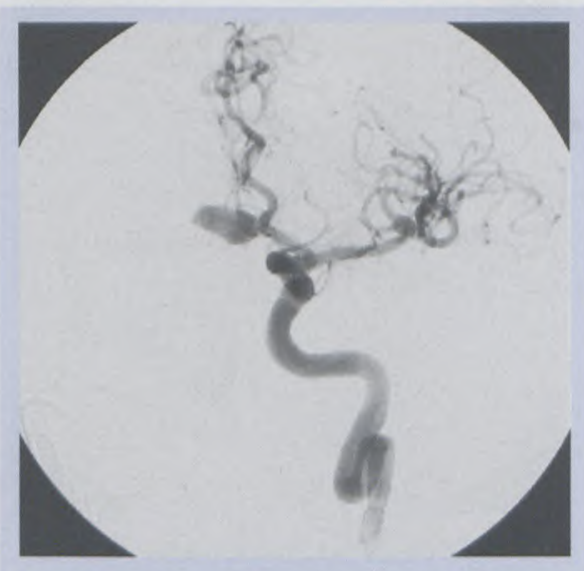

Fig. 2b. Selective left intemal carotid DSA image showing the anterior communicating complex aneurysm.

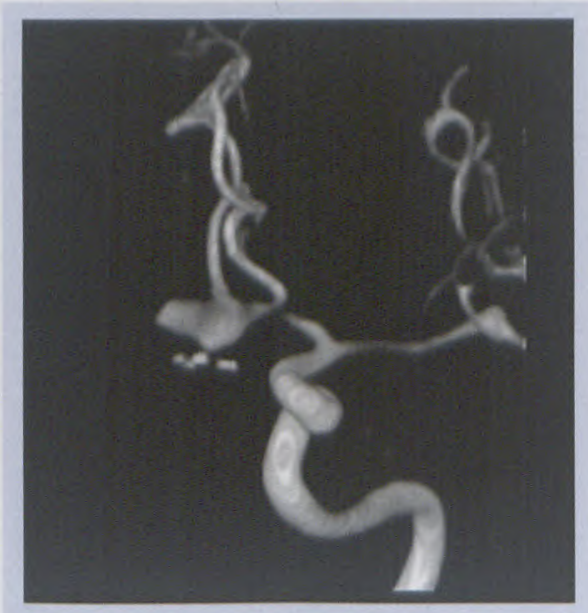

Fig. 2c. First 3D reconstruction 


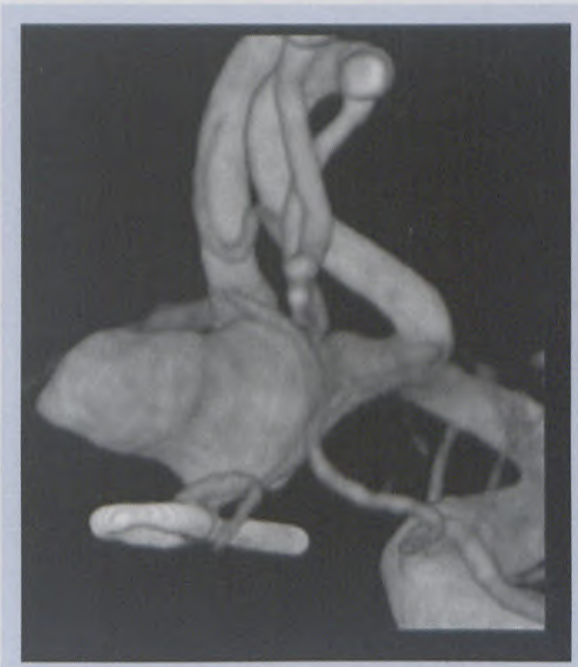

Fig. 2d. Second reconstruction. Note the surgical clip placed some 14 years prior for treatment of an aneurysm at the same site, indicating local recurrence of this aneurysm.

computer that does this is attached to its own separate workstation with a high-resolution monitor. The first reconstructed volume shows an image of the vessels within the entire area examined. The 3D image can then undergo further reconstructions to produce higher resolution images of selected parts of the initial image (Figs $1,2)$. These images can then be rotated in any plane on the workstation to allow one to view a lesion from any direction, including projections impossible to obtain by the $\mathrm{C}$-arm itself and including views from above and below the lesion. A lesion, such as a cerebral aneurysm and its adjacent parent vessel, can be 'cut-open' and viewed from within. The real time virtual endovascular view allows one to travel through the vessel lumen to visualise it from within. Although visually impressive we have found this to be of little practical value. A more valuable tool is that measurements of a lesion or vessel diameter can be made without the need for any calibration against an external object. These measurements are consistent
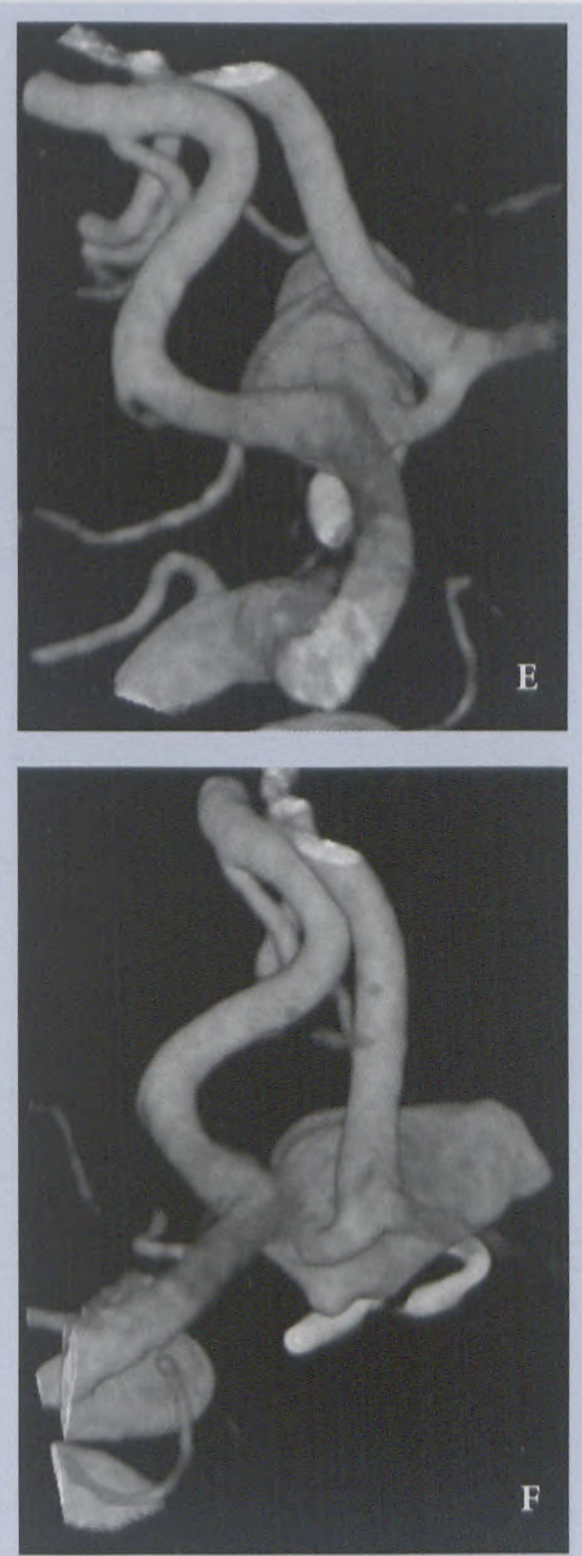

Fig. $2 e, 2 f$. The relationships of the left $A 1$ and $A 2$ vessels, the anterior communicating artery and the right $A 2$ vessel are clearly shown from behind (Fig .2e) and posterolaterally (Fig. 2f).

and highly accurate, assuming that the machine is properly and routinely calibrated, and are accurate to within $2 \%$ of actual size. Images can be stored as individual snapshots such as in JPEG format, as unidirectional rotational movies in AVI format, or as interactive virtual reality files in VRML format. These can be written to a CD for distribution and playback on remote computers. Hard copies of images are made by a laser printer attached to the workstation.

\section{Local results}

Between 1 January 2002 and 31 December 2002 we performed 64 3D rotational angiographic studies on 59 patients at the Unitas Interventional Unit. Two studies were non-neurological, 1 involved a knife injury of a vertebral artery and 1 involved a cerebral arteriovenous malformation. Of the remaining 55, 21 (38\%) underwent endovascular treatment of intracranial aneurysms including 3 stentassisted cases. In 2 cases coil placement was deemed feasible based upon the 3DRA images but we were unable to achieve stability of the initial coil placed within the aneurysm resulting in abortion of the attempted endovascular treatment. We have found that it is difficult to appreciate the true morphology of very small aneurysms ( $<4 \mathrm{~mm}$ ) which can have broader necks than are suggested by the 3DRA studies. This pitfall may in part be related to the choice of window settings used when evaluating such small aneurysms conferring an element of operator dependance on the system. ${ }^{6}$ On at least two occasions we found small coincidental aneurysms not detected during conventional 2D DSA studies.

\section{Benefits of 3DRA}

More than ninety per cent of 3DRA studies in our unit were done in order to demonstrate the architecture of cerebral aneurysms. We performed one 3D renal angiogram, one cerebral AVM study, one extracranial vertebral arterial study (Fig. 3) and one intrahepatic cholangiogram during this period, but the principal 


\section{ORIGINAL ARTICLE}

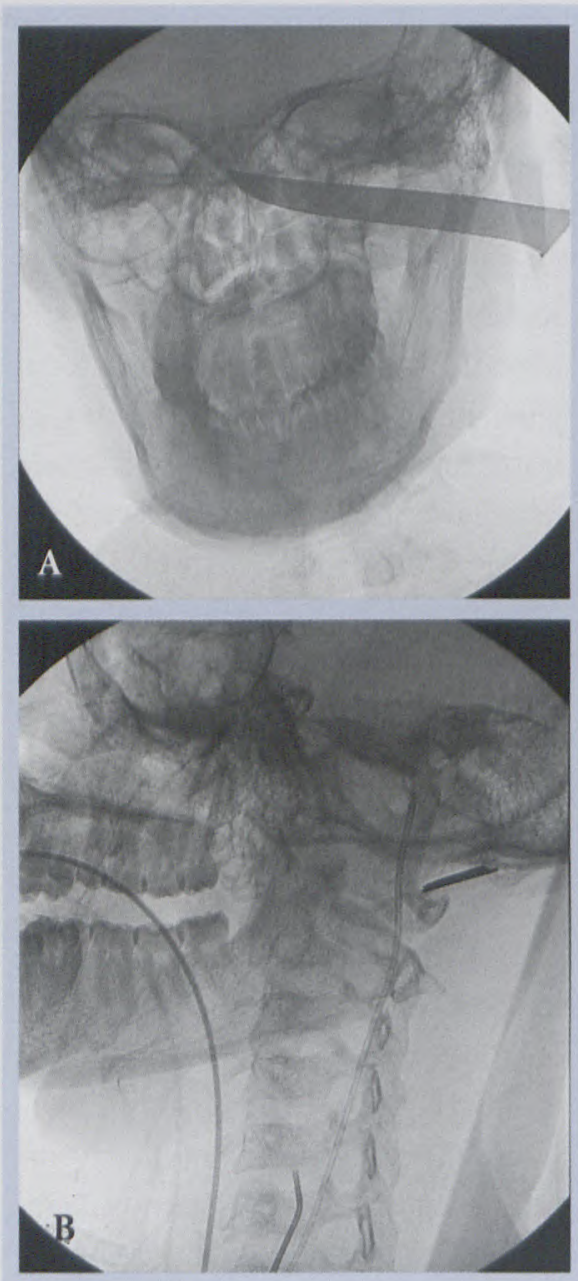

Figs. 3a, b. A knife blade is embedded below the left mastoid process and above the posterior arch and left lateral mass of $\mathrm{C1}$.

application remains the study of cerebral aneurysms. We are now able to demonstrate a cerebral aneurysm more accurately from the point of view of clearer angioarchitecture and improved demonstration of its relationship to its parent and adjacent arteries, particularly in difficult anatomic areas such as the anterior communicating artery complex, and middle cerebral bifurcation and trifurcation together with any associated developmental arterial anomalies (Figs 2, 4). Our standard protocol during cerebral angiography for aneurysms is to perform PA, lateral and superior oblique standard pro-
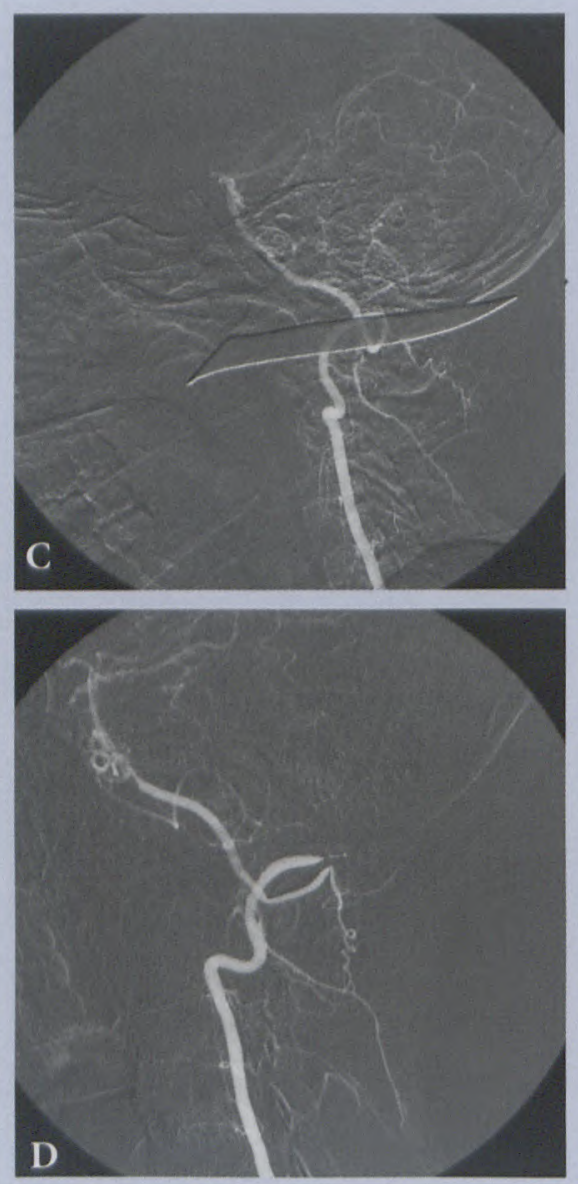

Figs $3 c$, $d$. Selective left vertebral DSA runs show the vertebral artery "draped" around the blade.

jections and if an aneurysm is identified to precede immediately to 3DRA. This cuts down on the performance of runs in multiple projections to try and identify the neck of the aneurysm thereby reducing the amount of contrast used and the radiation dose during the diagnostic procedure. From the 3DRA image the best possible angiographic angle at which the aneurysm neck can be demonstrated is estimated which is termed the 'stand' view, or as we call it, the 'working' projection. A final conventional angiographic run can be performed in this working projection to show the aneurysm neck if needed. This is usually the projection used when endovascular coiling of an aneurysm is performed as it provides the clearest
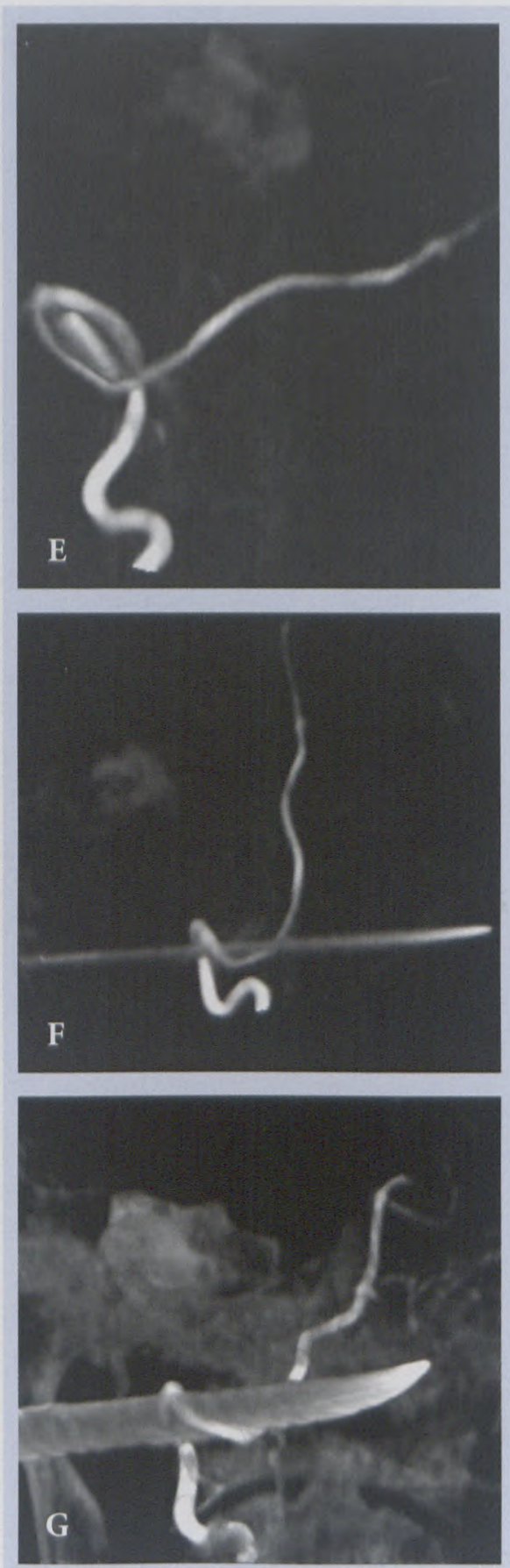

Figs $3 e, f, g$. 3DRA images show the left vertebral artery wound around the blade as it exits the left foramen transversarium and enters the dura at $\mathrm{C1}$.

view of the neck to check for coil prolapse out of the aneurysm lumen during coil placement.

Feedback from the local neurosurgical fraternity has to date been far less encouraging in that the provision of these high-resolution 3D images has 


\section{ORIGINAL ARTICLE}

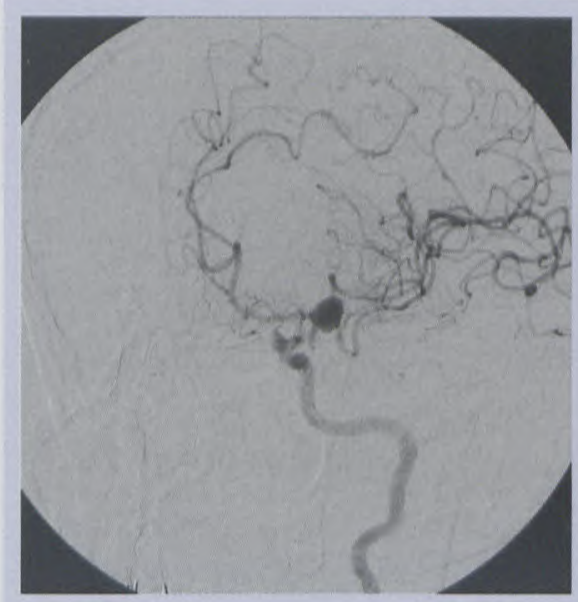

Fig. 4a. A large left middle cerebral bifurcation aneurysm.

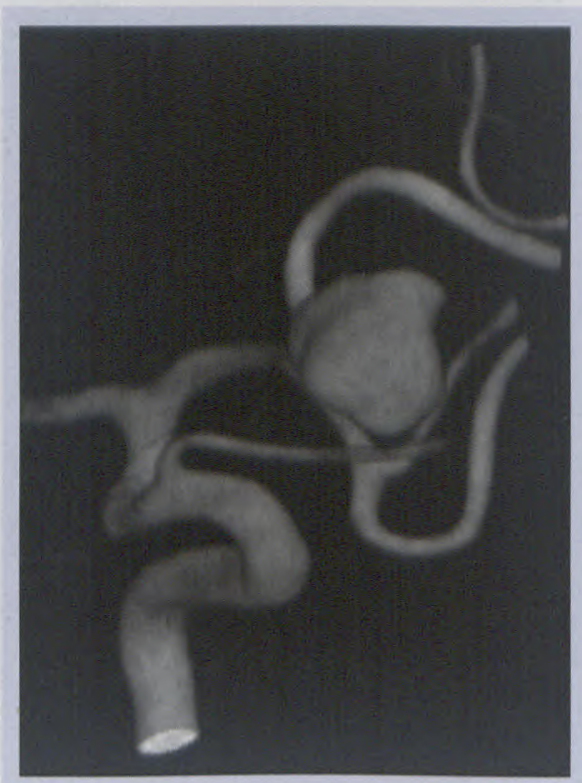

Fig. 4b. When seen from the front its relationship to the adjacent M2 branches is unclear.

generally not altered the pre-surgical planning of operative cases. Of more importance to the neurosurgeon is the external enviroment around an aneurysm including the adjacent softtissue and bony structures rather than detail of the internal structure of an aneurysm, although information about the size, direction of the fundus and neck morphology are as important in surgical planning as is knowledge of the adjacent arterial structures. This attitude may, however, also

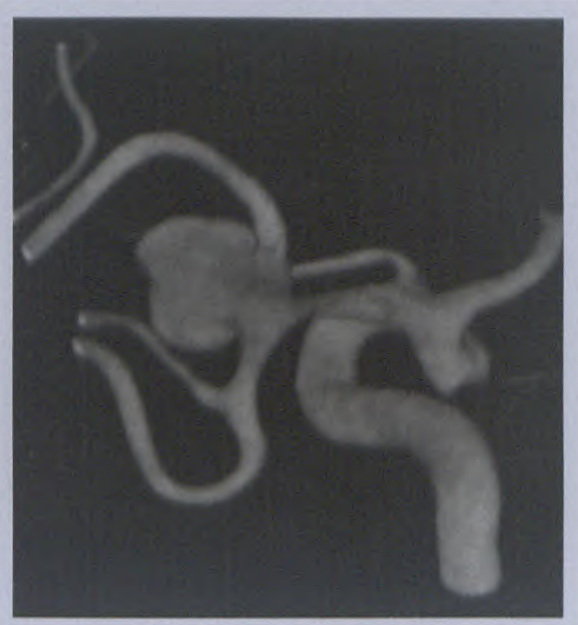

Fig. 4c. When seen from behind this becomes very clear.

largely reflect a lack of exposure by many local neurosurgeons to 3DRA images at this stage and may change in the future as in other parts of the world where such imaging has been shown to be of great benefit to the neurosurgeon in pre-surgical assessment. ${ }^{8}$ Thus the single greatest benefit of 3DRA in our experience has been assisting the endovascular treatment of cerebral aneurysms.

For the neurointerventionalist, knowledge of the internal architecture of any aneurysm is paramount. The neck-to-body ratio is crucial for predicting the stability of coils within the aneurysm lumen, and knowledge of the presence of branch vessel origins incorporated within the base or body of the aneurysm is also vital. The enhanced visualisation and more accurate measurements allow for a better selection of the type and size of coils to be placed as well as the type of microcatheter system to be used in advance, which has led to definite cost saving due to less wastage caused by improper judgement from the onset. Our 3DRA capability initially backfired on us as we began making very conservative judgements based upon perceived difficulties that could be encountered with certain aneurysm types and shapes, with the result that some aneurysms that could probably have been coiled were not. However, having undertaken a steep learning curve which partly coupled with a bolder approach to the endovascular treatment of wide-necked and complex aneurysms, has resulted in our treating over one-third of all aneurysms seen presently in our unit by endovascular means. This 'aggressive' approach now also employs balloon-remodelling and stent-scaffolding-assisted techniques all of which can be planned more efficiently with 3DRA (Fig. 5). 3DRA has also allowed us to predict more accurately those aneurysms which we cannot or should not do endovascularly, either due to unfavourable anatomy or to the knowledge that the long-term outcome for certain aneurysms would be better if managed surgically. Previously this would have entailed at least the use of a microcatheter and microwire to catheterise the aneurysm lumen and performance of a highrisk 'aneurysmogram' to assess the internal architecture of an aneurysm.

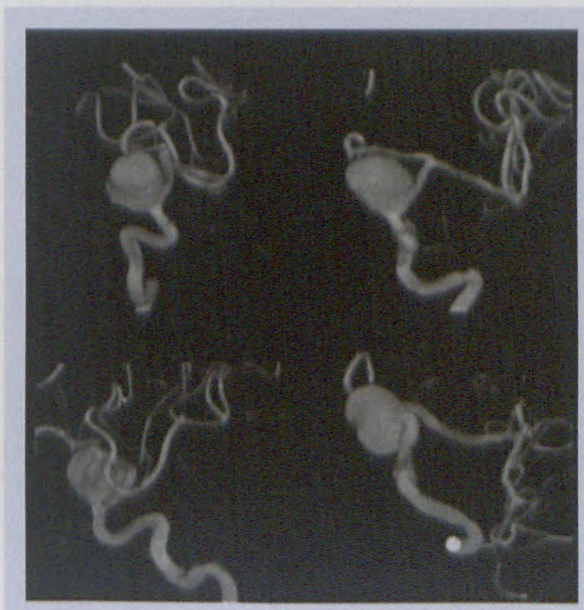

Fig. 5a. 3DRA images show a giant supracavemous aneurysm causing ipsilateral progressive vision loss. 


\section{ORIGINAL ARTICLE}

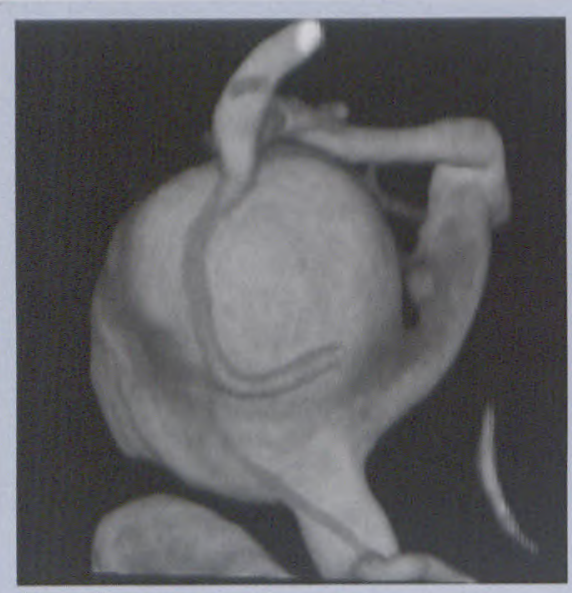

Fig. 5b. Volume-rendered image.

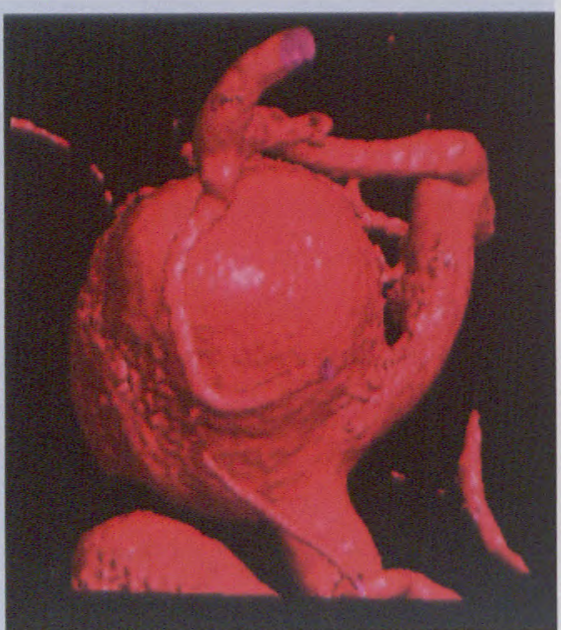

Fig. 5c. Surface-shaded display image.

Ultimately one or two coils (at a cost of around R5 000 each) might need to have been placed without detachment into the aneurysm lumen to assess coil stability and suitability for full packing. Often only having reached this stage did we decide that endovascular treatment was not feasible, abandoning the procedure having incurred considerable costs for no real gain. Now with 3DRA we are able to predict and select cases that are more suitable (or not) for endovascular treatment with a higher degree of success at the onset, which results in considerably less procedure-related risk to patients and definite cost savings to patients and funders primarily by avoiding

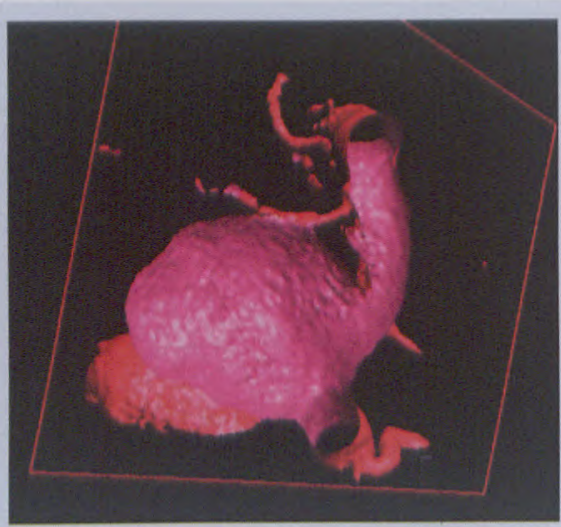

Fig. 5d. The aneurysm is 'cut open' to reveal detail of the afferent and efferent vessels. This allows planning of stent placement between the two.

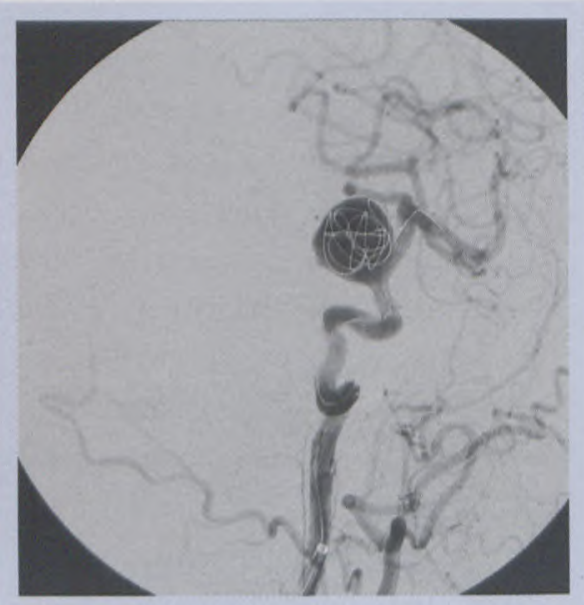

Fig. 5e. Following stent placement across the neck of the aneurysm to ensure patency of the carotid artery the remainder of the aneurysm is packed with coils.

these unecessary procedures. During coil placement (or other endovascular procedures) accurate measurement of the aneurysm or adjacent vessel dimensions allows for the correct choice of coil, balloon or stent size, also reducing wastage resulting from inaccurate planning.

As shown by both ourselves and Heautot et al., ${ }^{6}$ on occasion small aneurysms missed with conventional 2D DSA studies may be found on 3DRA studies. Although these small aneurysms are mainly incidental findings often seen by chance as they fall into the same field of view as another

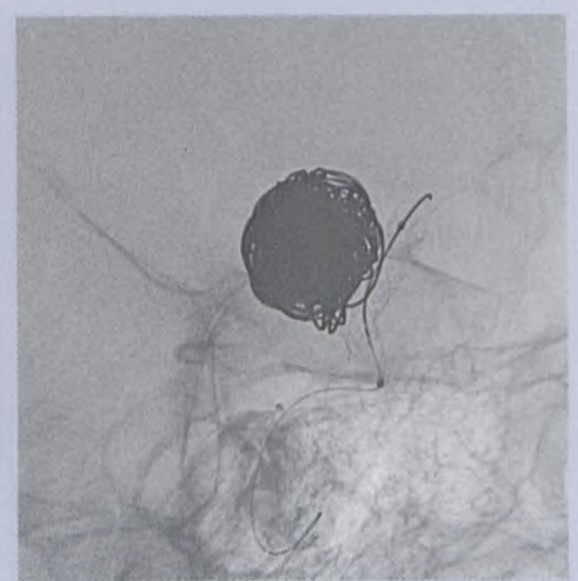

Fig. 5f. The stent is barely visible adjacent to the coils.

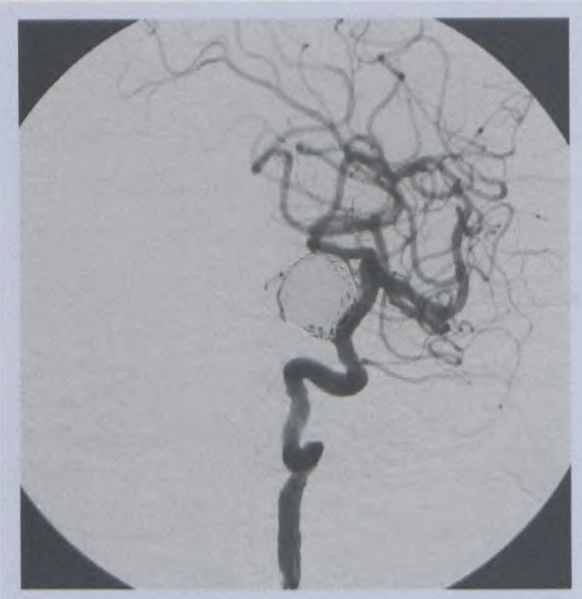

Fig. 5g. After coiling of the aneurysm good flow is noted through the stent adjacent to the coil mass with no filling of the adjacent aneurysm.

target lesion, and given the $2-27 \%$ negative aneurysm yield in angiograms done soon after subarachnoid haemorrhage $(\mathrm{SAH})$ and the poor sensitivity of CT and MR angiography in the detection of aneurysms $<3 \mathrm{~mm}$ in size, ${ }^{12}$ this raises the question about the potential deliberate use of 3DRA for the detection of very small or angiographically 'occult' aneurysms in this setting. To date we have only used 3DRA for the assessment of known aneurysms or those already identified with 2D DSA. Given the very high spatial and contrast resolution of 3DRA it is possible that such 
aneurysms may be detected where 3DRA is used for examining both internal carotid and the vertebrobasilar territories in cases where conventional 2D DSA fails to show a causative aneurysm in the setting of acute SAH.

Other potential uses for 3DRA, some of which we ourselves have not yet explored, include the assessment of cerebral and spinal malformations, extracranial carotid (and vertebral) artery disease and aortic aneurysms prior to stent or stent-graft placement. That 3DRA capabilities are becoming indispensable in current angiographic practice is attested to by the fact that most major manufacturers are now producing their newest angiographic units with 3DRA packages as standard fixtures and no longer as optional extras.

\section{Conclusion}

In many parts of the world catheter-based diagnostic angiography is progressively being replaced by non-invasive sectional imaging methods, often resulting in three-dimensional representations of the data thus acquired. In cerebrovascular disease although many lesions can be well demonstrated with CT or MR angiography, the three-dimensional images obtained with these modalities either provide too much extraneous information or too little detailed data about the lesion itself. Cerebral 3DRA has been shown to be of benefit in demonstrating the anatomy and relationships of cerebral aneurysms prior to either endovascular or surgical treatment. Advantages for the endovascular therapist include better patient selection and thus prediction of success prior to embarking upon any invasive treatment, as well as enhanced safety and cost-effectiveness due to more accurate pre-procedural planning for those cases treated this way. The possible role for 3DRA in other vascular pathologies remains to be defined.

\section{References}

1. Voight K, Stoeter P, Petersen D. Rotational cerebral roentgenography. 1: Evaluation of the technical procedure and diagnostic application with model studies. Neuroradiology 1975; 10: 95-100.

2. Hoff DJ, Wallace C, TerBrugge KG, Gentili F. Rotational angiography assessment of cerebral aneurysms. Am J Neuroradiol 1994; 15: 19451948.

3. Tu RK, Cohen WA, Maravilla KR, et al. Digital subtraction rotational angiography for aneurysms of the intracranial anterior circula- tion: Injection method and optimization. Am J Neuroradiol 1996; 17: 1127-1136.

4. Turjman F, Bendib K, Girerd C, Froment JC, Amiel M. Pretherapeutic evaluation of intracranial aneurysms using three-dimensional angiography (3D morphometer): preliminary results. In: Taki W, Picard L, Kikuchi H, eds. Advances in Interventional Neuroradiology and Intravascular Neurosurgery. Amsterdam: Elsevier Science BV: 1996: 75-79.

5. Fahrig R, Fox AJ, Lownie S, Holdsworth DW. Use of a C-arm system to generate true three-dimensional computed rotational angiograms: Preliminary in vitro and in vivo results. $A m \mathrm{~J}$ Neuroradiol 1997; 18: 1507-1514.

6. Heautot JF, Chabert E, Gandon Y, et al. Analysis of cerebrovascular diseases by a new 3 dimensional computeri zed X-ray angiography system. Neuroradiology 1998; 40: 203-209.

7. Ishihara S, Ross IB, Piotin M, Weill A, Aerts H, Moret J. 3D Rotational angiography: Recent experience in the evaluation of cerebral aneurysms for treatment. Interventional Neuroradiology 2000; 6: 85-94.

8. Tanoue S, Kiyosue H, Kenai H, Nakamura $\mathrm{T}$, Yamashita M, Mori $\mathrm{H}$. Three-dimensional reconstructed images after rotational angiography in the evaluation of intracranial aneurysms : Surgical correlation. Neurosurgery 2000; 47: 866871.

9. Anxionnat R, Bracard S, Ducrocq X, et al. Intracranial aneurysms: Clinical value of $3 \mathrm{D}$ digital subtraction angiography in the therapeutic decision and endovascular treatment. Radiology 2001; 218: 799-808.

10. Abe T, Hirohata M, Tanaka N, et al. Clinical benefits of rotational 3D angiography in endovascular treatment of ruptured cerebral aneurysm. Am JNeuroradiol 2002; 23: 686-688.

11. Klucznik RP. Current technology and clinical application of three-dimensional angiography. Radiological Clinics of North America 2002; 40: 711-728.

12. White PM, Wardlaw JM, Easton V. Can noninvasive imaging accurately depict intracranial aneurysms ? A systematic review. Radiology 2000; 217: 316-370 